\title{
Dynamical Franz-Keldysh Effect
}

\author{
Jauho, Antti-Pekka; Johnsen, Kristinn
}

Published in:

Physical Review Letters

Link to article, DOI:

10.1103/PhysRevLett.76.4576

Publication date:

1996

Document Version

Publisher's PDF, also known as Version of record

Link back to DTU Orbit

Citation (APA):

Jauho, A-P., \& Johnsen, K. (1996). Dynamical Franz-Keldysh Effect. Physical Review Letters, 76(24), 45764579. https://doi.org/10.1103/PhysRevLett.76.4576

\section{General rights}

Copyright and moral rights for the publications made accessible in the public portal are retained by the authors and/or other copyright owners and it is a condition of accessing publications that users recognise and abide by the legal requirements associated with these rights.

- Users may download and print one copy of any publication from the public portal for the purpose of private study or research.

- You may not further distribute the material or use it for any profit-making activity or commercial gain

- You may freely distribute the URL identifying the publication in the public portal

If you believe that this document breaches copyright please contact us providing details, and we will remove access to the work immediately and investigate your claim. 


\title{
Dynamical Franz-Keldysh Effect
}

\author{
A. P. Jauho and K. Johnsen \\ Mikroelektronik Centret, Technical University of Denmark, Bldg. 345 East, DK-2800 Lyngby, Denmark
}

(Received 7 December 1995)

\begin{abstract}
We introduce and analyze the properties of dynamical Franz-Keldysh effect, i.e., the change of density of states, or absorption spectra, of semiconductors under the influence of time-dependent electric fields. In the case of a harmonic time dependence, we predict the occurrence of significant fine structure, both below and above the zero-field band gap, which should be experimentally observable. [S0031-9007(96)00436-X]
\end{abstract}

PACS numbers: 71.15.Cr, 78.20.Bh, 78.20.Jq, 78.47.+p

Almost forty years ago Franz [1] and Keldysh [2] pointed out that static electric fields modify the linear optical properties of bulk semiconductors near the optical absorption edge: The absorption coefficient becomes finite (though small) for photon energies below the band gap, and the absorption coefficient shows oscillations for energies above the band gap. Studies of this kind regained importance with the development of quantum confined semiconductor structures, and a large body of both experimental and theoretical knowledge has been developed since the mid 1980s [3]. The purpose of this Letter is to point out that the absorption curve develops additional structure when the sample is placed in a strong ac field, such as generated by a free-electron laser. As we shall demonstrate below, the zero-field density of states splits into replicas, with field-dependent shifts and time-dependent amplitudes. In contrast to the static case, where it is sufficient to solve a simple Schrödinger equation, the time-dependent case necessitates the use of a more advanced formalism; in the present case we apply nonequilibrium Green function techniques. In order to present our predictions in a clear-cut manner, we have stripped the model calculation from complications that may be present in real samples, such as scattering due to sample nonidealities and phonons or excitonic effects. Thus, the main motivation of the present analytic study is to point out the existence of an effect, and not address the quantitative details, which can be treated properly only with a full-scale numerical calculation based, for example, on the solution of the semiconductor Bloch equations [4].

The calculation of the electroabsorption coefficient $\alpha(\omega)$ for free carriers is discussed in textbooks [5], and the result can be expressed as

$$
\alpha(\omega)=\alpha_{0} \sum_{n}\left|\psi_{n}(\mathbf{r}=0)\right|^{2} \delta\left(E_{n}-\hbar \omega\right),
$$

where $\psi_{n}$ and $E_{n}$ are the eigenfunctions and eigenvalues of the electron-hole pair Schrödinger equation, respectively. The important feature of Eq. (1) for our purposes is that it states that the absorption coefficient is essentially given by the joint density of states, $\alpha(\omega) \simeq \rho(\omega) \equiv \sum_{n} \delta(\omega-$ $\left.E_{n}\right)$. More rigorously, the absorption coefficient can be evaluated within the framework of the equilibrium many- body theory [6]. There, the absorption coefficient (for noninteracting carriers) is expressed as a convolution between the imaginary parts of the retarded Green functions for electrons and holes or, equivalently, by their spectral functions. The important question is then: Can one perform a similar analysis in time-dependent situations? The answer is provided by the nonequilibrium theory of Kadanoff and Baym [7] and Keldysh [8]: In nonequilibrium the diagrammatic structure of the perturbation theory is the same as in equilibrium, with the proviso that real-time integrals must be generalized to a complex contour [9]. Thus, the nonequilibrium spectral function can be calculated from a Dyson equation which is formally identical to the equilibrium case, and one can analyze the electroabsorption properties of a semiconductor in an applied time-dependent electric field by evaluating [10]

$$
\begin{aligned}
\rho(\omega, T) & =\frac{i}{2 \pi} \sum_{\mathbf{k}}\left[\tilde{G}^{r}(\mathbf{k}, \omega, T)-\tilde{G}^{a}(\mathbf{k}, \omega, T)\right] \\
& =\frac{1}{2 \pi} \sum_{\mathbf{k}} \tilde{A}(\mathbf{k}, \omega, T) .
\end{aligned}
$$

Equation (2) introduces ageneralized density-of-states function, which is time dependent due to the external electric field. Whether a measurement probes $\rho(\omega, T)$ or perhaps its suitably weighted time average depends on the various time scales of the particular experimental setup, here we focus on the full time dependence, because it contains the most information [11]. In (2) we also introduce the gauge-invariant form $\tilde{G}^{r}(\mathbf{k}, \omega, T)$ of the two-time Green function $G^{r}\left(\mathbf{x}, \mathbf{x}^{\prime}, t, t^{\prime}\right)$, which we calculate via its Dyson equation. Effects due to interactions can be taken into account by introducing a suitable self-energy. Under certain conditions, the Dyson equation for the retarded Green function may involve particle densities; in this case, one must also solve the appropriate quantum kinetic equation for the distribution function. However, this problem is not addressed in the present paper.

In an actual calculation the longitudinal electric field must be introduced by using some explicit gauge; however, predictions for observables must clearly be gauge independent, and hence a gauge-invariant formulation 
is essential. In general, the transformation to gaugeinvariant variables occurs via [12]

$$
\begin{aligned}
\tilde{G}^{r}(p, X)= & \int d^{4} q G^{r}(q, X) \\
& \times \exp \left(i q_{\mu}\left[p_{\mu}+\int_{-1 / 2}^{1 / 2} d \lambda A_{\mu}(X+\lambda q)\right]\right),
\end{aligned}
$$

where we have used the Einstein summation convention and a "covariant" notation, where four-momenta and coordinates are given by $p \equiv(\omega, \mathbf{k}), q \equiv(\tau, \mathbf{r})=(t-$ $\left.t^{\prime}, \mathbf{x}-\mathbf{x}^{\prime}\right)$, and $X \equiv(T, \mathbf{R})=\left(\left[t+t^{\prime}\right] / 2,\left[\mathbf{x}+\mathbf{x}^{\prime}\right] / 2\right)$. Correspondingly, the potentials defining the electric field are contained in $A \equiv(\phi, \mathbf{A})$.

In our calculation of the density of states for a uniform but time-dependent field, it is convenient to describe the electric field with a vector potential, $\mathbf{A}(t)=$ $-\mathbf{E} \sin (\Omega t) / \Omega$ (here we restrict ourselves to harmonic time dependence). The retarded Green function satisfies [13]

$$
\left\{i \frac{\partial}{\partial t}-\epsilon[\mathbf{p}-\mathbf{A}(t)]\right\} G^{r}\left(\mathbf{p}, t, t^{\prime}\right)=\delta\left(t-t^{\prime}\right) .
$$

Using (3), Eq. (4) immediately leads to the gaugeinvariant spectral function $\tilde{A}=i\left(\tilde{G}^{r}-\tilde{G}^{a}\right)$,

$$
\begin{aligned}
\tilde{A}(\mathbf{k}, \omega, T)= & \int d \mathbf{r} d \tau e^{i w} \int d \mathbf{p} e^{i \mathbf{r} \cdot \mathbf{p}} \\
& \times \exp \left\{-i \int_{T-\tau / 2}^{T+\tau / 2} d t_{1} \epsilon\left[\mathbf{p}-\mathbf{A}\left(t_{1}\right)\right]\right\}
\end{aligned}
$$

where

$$
w=\tau \omega-\mathbf{r} \cdot \mathbf{k}-\int_{T-\tau / 2}^{T+\tau / 2} \frac{d t_{1}}{\tau} \mathbf{r} \cdot \mathbf{A}\left(t_{1}\right) .
$$

It is illustrative to see how the static field results [14] can be recovered from Eqs. (5) and (6). In the limit $\Omega \rightarrow 0$ and for parabolic bands Eq. (5) reduces to

$$
\begin{aligned}
& A_{\mathrm{dc}}(\mathbf{k}, \tau)=\exp \left[-i\left(\epsilon_{k} \tau+E^{2} \tau^{3} / 24\right)\right], \\
& A_{\mathrm{dc}}(\mathbf{k}, \omega)=(2 \pi / \beta) \operatorname{Ai}\left[\left(\epsilon_{k}-\omega\right) / \beta\right],
\end{aligned}
$$

with $\beta=\left(\hbar^{2} e^{2} E^{2} / 8 m\right)^{1 / 3}$, and $\operatorname{Ai}(x)$ is the Airy function. Evaluation of the momentum sum then leads in three dimensions to

$$
\rho_{\mathrm{dc}}^{3 \mathrm{D}}(\omega) \propto\left\{\mathrm{Ai}^{2}[-(\omega / \Theta)]+(\omega / \Theta) \mathrm{Ai}^{2}[-(\omega / \Theta)]\right\},
$$

with $\Theta=4^{1 / 3} \beta / \hbar$. Equation (8) is the standard form for the Franz-Keldysh line shape [5].

In the time-dependent case, one finds

$$
\begin{gathered}
\tilde{A}(\mathbf{k}, \omega, T)=\int d \tau \cos \left[\left(\omega-\epsilon_{k}-\omega_{F}\right) \tau+X(\tau)\right. \\
+2 Y(\tau)],
\end{gathered}
$$

where we have introduced the notation

$$
\begin{aligned}
& X(\tau)=\frac{\omega_{F}}{\Omega} \sin \Omega \tau \cos 2 \Omega T, \\
& Y(\tau)=\frac{\omega_{F}}{\Omega} \frac{4 \sin ^{2}(\Omega \tau / 2) \sin ^{2} \Omega T}{\Omega \tau}
\end{aligned}
$$

and defined the field parameter $\omega_{F}=e^{2} F^{2} / 4 m \hbar \Omega^{2}$ [15]. An explicit analytic evaluation of the integral in (9) is not possible, nor is a straightforward numerical calculation feasible due to its singular character. The singularities, however, can be isolated with analytic means, and we proceed as follows. The trigonometric identity $\cos (x+$ $y)=\cos x-2 \sin (x+y / 2) \sin (y / 2)$ allows us to write (9) as a sum of two terms, $\tilde{A}=\tilde{A}_{1}+\tilde{A}_{2}$, where

$$
\begin{gathered}
\tilde{A}_{1}(\mathbf{k}, \omega, T)=\int d \tau \cos \left[\left(\omega-\epsilon_{k}-\omega_{F}\right) \tau+X(\tau)\right] \\
\tilde{A}_{2}(\mathbf{k}, \omega, T)=-2 \int d \tau \sin \left[\left(\omega-\epsilon_{k}-\omega_{F}\right) \tau\right. \\
+X(\tau)+Y(\tau)] \sin Y(\tau) .
\end{gathered}
$$

The integral defining $\tilde{A}_{2}$ is convergent, and its numerical evaluation is straightforward. On the other hand, analytic progress can be made with $\tilde{A}_{1}$, which contains the aforementioned singularities. Recalling the identity $\int_{-\infty}^{\infty} d x \cos (a x-b \sin x)=2 \pi \sum_{n=-\infty}^{\infty} J_{n}(b) \delta(n-$ $a$ ), where $J_{n}$ is the $n$th order Bessel function, we finally obtain

$$
\begin{aligned}
\tilde{A}_{1}(\mathbf{k}, \omega, T)= & 2 \pi \sum_{n=-\infty}^{\infty} J_{n}\left[-\frac{\omega_{F}}{\Omega} \cos (2 \Omega T)\right] \\
& \times \delta\left(\omega-\epsilon_{k}-n \Omega-\omega_{F}\right) .
\end{aligned}
$$

These two components, $\tilde{A}_{1}$ and $\tilde{A}_{2}$, of the spectral function have quite distinct properties. The frequency sum rule is exhausted by $\tilde{A}_{1}$ alone, i.e., $\int d \omega / 2 \pi \tilde{A}_{1}(\omega, T)=1$, while $\int d \omega / 2 \pi \tilde{A}_{2}(\omega, T)=0$. Further, the delta-function singularities of $\tilde{A}_{1}$ are reminiscent of photonic sidebands [16]; however, in the present case these features are shifted by the field- and frequencydependent parameter $\omega_{F}$. In the zero-field limit, $\tilde{A}_{1}$ reduces to the field-free result $a_{0}(k, \omega)=2 \pi \delta\left(\omega-\epsilon_{k}\right)$, while $\tilde{A}_{2}$ vanishes. Note also that the spectral function depends only on even powers of the electric field. This is consistent with physical intuition: Since there is no preferred direction, it does not matter if the field direction is reversed, $\mathbf{E} \rightarrow-\mathbf{E}$. Finally, the spectral function is negative in parts of the $(\omega, T)$ plane: This reflects the nonequilibrium nature of the present problem. An important property of Eq. (9) is that it leads to a positive semidefinite $\rho(\omega, T)$; this justifies the nomenclature "generalized density of states." The functions $\tilde{A}_{1}$ and $\tilde{A}_{2}$ are displayed in Figs. 1 and 2, respectively. We 


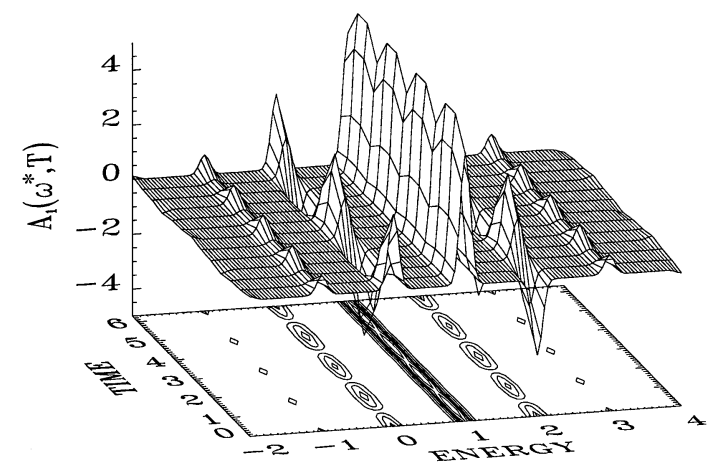

FIG. 1. The function $\tilde{A}_{1}$ as a function of the variable $\omega^{*}=$ $\left(\omega-\epsilon_{k}\right) / \Omega$. The field strength is given by $\omega_{F} / \Omega=1$. The delta-function singularities of $\tilde{A}_{1}$ have been broadened for illustrative purposes. In addition to the time-dependent features, one should pay attention to the field-induced shift of the main feature from $\omega=\epsilon_{k} \rightarrow \epsilon_{k}+\omega_{F}$.

can use the above results to calculate a time-dependent density-of-states function $\rho(\omega, T)$. The contribution arising from $\tilde{A}_{1}$ is readily evaluated by using the delta function, while $\tilde{A}_{2}$ requires some more work. In three dimensions we find

$$
\begin{aligned}
\rho_{1}^{3 \mathrm{D}}(\omega, T)= & \frac{\left(2 m^{3}\right)^{1 / 2}}{2 \pi^{2} \hbar^{5 / 2}} \sum_{n=-\infty}^{\left[\frac{\omega-\omega_{F}}{\Omega}\right]} J_{n}\left[-\frac{\omega_{F}}{\Omega} \cos (\Omega T)\right] \\
& \times\left(\omega-\omega_{F}-n \Omega\right)^{1 / 2}
\end{aligned}
$$

and

$$
\begin{aligned}
\rho_{2}^{3 \mathrm{D}}(\omega, T)= & \frac{\left(\pi m^{3}\right)^{1 / 2}}{4 \pi^{3} \hbar^{5 / 2}} \int_{0}^{\infty} \frac{d \tau}{\tau^{3 / 2}} \sin [Y(\tau)] \\
& \times \sin \left[\left(\omega-\omega_{F}\right) \tau\right. \\
& \quad+X(\tau)+Y(\tau)+\pi / 4]
\end{aligned}
$$

Here the summation over $n$ should be cut off at the largest integer not exceeding $\left(\omega-\omega_{F}\right) / \Omega$. A numerical evaluation [11] shows that Eqs. (15) and (16) lead to an absorption curve, which is still dominated by the freeelectron-like square-root components generated by the photonic sidebands in $\tilde{A}_{1}$. Here we focus on the twodimensional case, where more dramatic effects are seen.

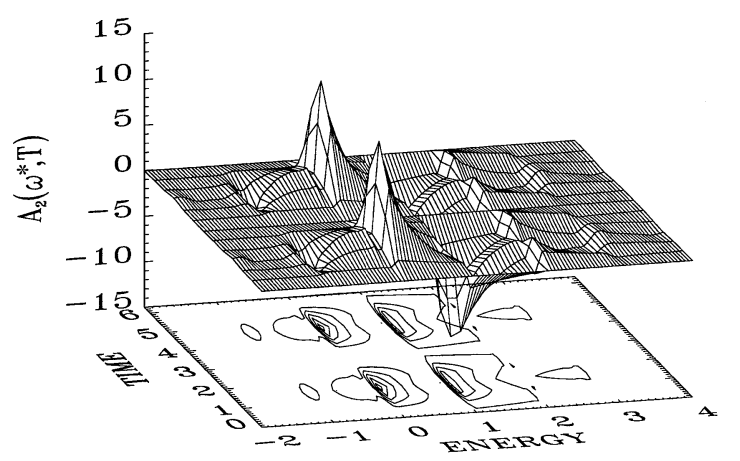

FIG. 2. The function $\tilde{A}_{2}$. The parameters are defined in the caption of Fig. 1.

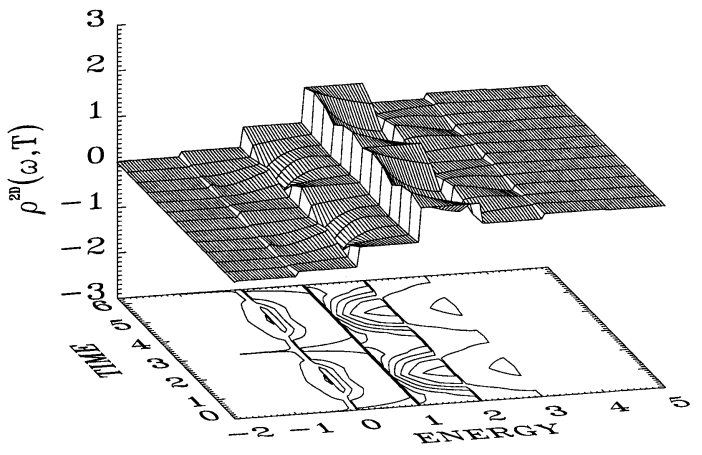

FIG. 3. Density of states, two dimensions.

One finds

$$
\begin{aligned}
\rho_{1}^{2 \mathrm{D}}(\omega, T)= & \frac{m}{2 \pi \hbar^{2}} \sum_{n=-\infty}^{\left[\frac{\omega-\omega_{F}}{\Omega}\right]} J_{n}\left[-\frac{\omega_{F}}{\Omega} \cos (\Omega T)\right] \\
& \times \theta\left(\omega-\omega_{F}-n \Omega\right), \\
\rho_{2}^{2 \mathrm{D}}(\omega, T)= & \frac{m}{2 \pi^{2} \hbar^{2}} \int_{-\infty}^{\infty} \frac{d \tau}{\tau} \sin [Y(\tau)] \\
& \times \cos \left[\left(\omega-\omega_{F}\right) \tau+X(\tau)+Y(\tau)\right],
\end{aligned}
$$

and a numerical example is shown in Fig. 3. The zerofield step function reflecting the two-dimensional density of states is split into several substeps extending to energies well below the gap, and the above threshold values are modified by additional structures, whose amplitudes tend to zero for increasing energies. The relative amplitude of the substeps changes as a function of time, and the fine structure in the energy dependence is due to $\tilde{A}_{2}$.

These modifications of the density of states, both below and above the zero-field absorption threshold, constitute the dynamical analog of the static Franz-Keldysh effect.

It is also of interest to examine how the time-dependent density of states depends on the field strength at a fixed frequency. The effects are most easily analyzed by examining the time average or the density of states (this would be appropriate for a cw measurement), $\rho_{\text {ave }}(\omega) \equiv$ $(\Omega / 2 \pi) \int_{0}^{2 \pi / \Omega} d T \rho(\omega, T)$, and Fig. 4 shows the results

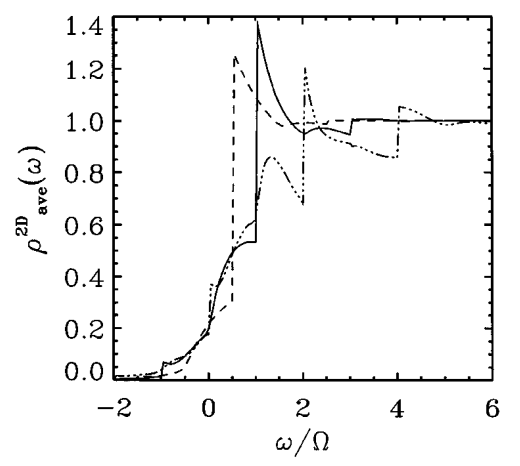

FIG. 4. Time-averaged two-dimensional density of states (in units of zero-field density of states) for $\omega_{F} / \Omega=0.5$ (dashed line), 1 (solid line), and 2 (dash-dotted line). 
of such a calculation [17]. The following features of $\rho_{\text {ave }}(\omega)$ are of importance: (i) The main absorption edge moves to higher energies with increasing field strength; (ii) there is a significant enhancement at the absorption edge; and (iii) the range of energies where the edge is modified increases with increasing field strengths [18].

Finally, let us comment on the observability of the predicted effects. There are several limiting factors: the spectral resolution in an absorption measurement, the available frequencies, and the maximal ac-field strengths that can be applied to the sample. From the results presented above, we conclude that the most favorable conditions are obtained if $\omega_{F} / \Omega>1$. It would appear that this condition is most easily achieved at low frequencies. However, a small $\Omega$ implies that all the fine structure would be confined to the immediate neighborhood of the absorption edge (in the example of Fig. 4 the fine structure extends only a few $\Omega$ 's above the edge), and not resolvable spectroscopically. Increasing $\Omega$ leads to an increased $\mathbf{E}$ in order to maintain sufficiently large $\omega_{F} / \Omega$. By using parameters attainable with free-electron lasers [19], $\Omega \simeq 1 \mathrm{THz}, \mathbf{E} \simeq 1 \mathrm{MV} / \mathrm{m}$, and the GaAs effective mass, we estimate that $\omega_{F} / \Omega \simeq 1$. This implies that the fine structure extends a few meV around the absorption edge, and should be observable.

In summary, we have calculated the density of states of free carriers in time-dependent fields, and find that a harmonically varying external field leads to a field and frequency dependent shift of the main absorption edge, giving rise to fine structure ("dynamical Franz-Keldysh effect") of observable magnitude.

We acknowledge useful comments from Professor H. Haug, Professor S. Koch, and Professor Jai Singh.

[1] W. Franz, Z. Naturforsch. Teil A 13, 484 (1958).

[2] L. V. Keldysh, Sov. Phys. JETP 34, 788 (1958).

[3] For a review, see S. Schmitt-Rink, D.S. Chemla, and D. A. B. Miller, Adv. Phys. 38, 89 (1989).

[4] Two recent examples are T. Meier et al., Phys. Rev. Lett. 73, 902 (1994); 75, 2558 (1995).

[5] See, e.g., H. Haug and S. W. Koch, Quantum Theory of the Optical and Electronic Properties of Semiconductors (World Scientific, Singapore, 1994), 3rd ed., Chap. 17.

[6] For a review, see, e.g., H. Haug and S. Schmitt-Rink, Prog. Quantum Electron. 9, 3 (1984).

[7] L.P. Kadanoff and G. Baym, Quantum Statistical Mechanics (Benjamin, New York, 1962).

[8] L. V. Keldysh, Sov. Phys. JETP 20, 1018 (1965).

[9] For general reviews of nonequilibrium Green functions see, for example, D. C. Langreth, in Linear and Nonlinear Electron Transport in Solids, edited by J. T. Devreese and
E. van Doren (Plenum, New York, 1976); J. Rammer and H. Smith, Rev. Mod. Phys. 58, 323 (1986).

[10] From now on, the spectral functions should be interpreted as joint spectral functions. In the parabolic band case studied here, the only modification is that the mass must be interpreted as the effective mass of the electron-hole pair, and the zero of the energy scale is set by the energy gap.

[11] A full nonequilibrium theory for electroabsorption [K. Johnsen and A.P. Jauho (unpublished)] shows that (i) the absorption coefficient $\alpha(\omega, T)$ is given by the generalized density of states $\rho(\omega, T)$, within corrections of the order of $\Omega / \omega_{L}$ (here $\Omega$ is the frequency of the applied longitudinal field, and $\omega_{L}$ is the frequency of the probe signal); this number is $\simeq 10^{-2}$ in the numerical example discussed in the text; and (ii) the correction terms vanish in a cw measurement.

[12] R. Bertoncini and A.P. Jauho, Phys. Rev. B 44, 3655 (1991).

[13] The analysis following Eq. (4) is independent of the number of spatial dimensions, however, one should be aware of the tacit assumption that the electric field lies in a direction in which there is translational invariance.

[14] The static results have been used extensively in the analysis of high-field quantum transport in semiconductors; see, e.g., A.P. Jauho, in Quantum Transport in Semiconductors, edited by D. K. Ferry and C. Jacoboni (Plenum, New York, 1992), pp. 141-168.

[15] The parameter $\omega_{F}$ has an appealing physical interpretation: $\hbar \omega_{F}$ is the time-averaged kinetic energy of a free particle with charge $e$ and mass $m$ in a harmonic electric field of frequency $\Omega$.

[16] There is a rapidly growing literature on mesoscopic structures, where photon sidebands play an important role. Recent examples include L. P. Kouwenhoven et al., Phys. Rev. B 50, 1994 (1994); A. P. Jauho, N. S. Wingreen, and Y. Meir, Phys. Rev. B 50, 5528 (1994); A. Grincwajg et al., Phys. Rev. B 52, 12168 (1995).

[17] The time average of $\tilde{A}_{1}$ can be related analytically: $\quad \tilde{A}_{1, \text { ave }}(\mathbf{k}, \omega)=2 \pi \sum_{n=-\infty}^{\infty} \delta\left(2 n \Omega-\omega+\epsilon_{k}+\right.$ $\left.\omega_{F}\right) J_{n}^{2}\left(\omega_{F} / 2 \Omega\right)$. This result bears a close resemblance to results obtained by Jonson [Phys. Rev. B 39, 5924 (1989)] and Iñarrea et al. [Phys. Rev. B 50, 4582 (1994)]. These authors were, however, concerned with the time-dependent properties of tunneling structures, where the spatial nonuniformity plays an essential role, and a quantitative comparison is not possible.

[18] The second article of Ref. [4] cited above has reported analogous absorption enhancement for below gap absorption for a superlattice; however, the strong excitonic features mask possible absorption edge shifts and/or enhancements predicted in this work.

[19] We use the parameters estimated by P.S.S. Guimarães et al., Phys. Rev. Lett. 70, 3792 (1993). 